\title{
Correlation between CSF biomarkers of Alzheimer's disease and global cognition in a psychogeriatric clinic cohort
}

\author{
Márcia Radanovic, (iD Carlos A. Oshiro, Thiago Q. Freitas, Leda L. Talib, Orestes V. Forlenza \\ Laboratório de Neurociências (LIM-27), Departamento e Instituto de Psiquiatria, Hospital das Clinicas, Faculdade de Medicina, Universidade \\ de São Paulo (USP), São Paulo, SP, Brazil.
}

\begin{abstract}
Objective: The relationship between biomarkers of amyloid-beta aggregation $\left(A \beta_{1-42}\right)$ and/or neurodegeneration (Tau protein) in cerebrospinal fluid (CSF) and cognitive decline is still unclear. We aimed to ascertain whether CSF biomarkers correlate with cognitive performance in healthy and cognitively impaired subjects, starting from clinical diagnoses.

Methods: We tested for correlation between CSF biomarkers and Mini-Mental State Examination (MMSE) scores in 208 subjects: 54 healthy controls, 82 with mild cognitive impairment (MCI), 46 with Alzheimer's disease (AD), and 26 with other dementias (OD).

Results: MMSE correlated weakly with all CSF biomarkers in the overall sample $(r=0.242$, $p<0.0006) . A \beta_{1-42}$ and MMSE correlated weakly in $\mathrm{MCl}(r=0.247, p=0.030)$, and moderately in OD $(r=0.440, p=0.027)$. $t-T a u$ showed a weak inverse correlation with MMSE in controls $(r=-0.284$, $p=0.043)$ and $\mathrm{MCl}(r=-0.241, p=0.036)$, and a moderate/strong correlation in OD $(r=0.665)$, $p=0.0003)$. $p$-Tau correlated weakly with MMSE in AD $(r=-0.343, p=0.026)$ and moderately in OD $(r=-0.540, p=0.0005)$. The $A \beta_{1-42} / p-T a u$ ratio had a moderate/strong correlation with MMSE in OD $(r=0.597, p=0.001)$.

Conclusion: CSF biomarkers correlated best with cognitive performance in OD. t-Tau correlated weakly with cognition in controls and patients with $\mathrm{MCl}$. In $\mathrm{AD}$, only $\mathrm{p}$-Tau levels correlated with cognitive performance. This pattern, which has been reported previously, seems to indicate that CSF biomarkers might not be reliable as indicators of disease severity.
\end{abstract}

Keywords: Mild cognitive impairment; dementia; Alzheimer's disease; cerebrospinal fluid biomarkers

\section{Introduction}

Alzheimer's disease $(A D)$ is the most common neurodegenerative cause of dementia, affecting more than 35 million people worldwide, with its prevalence expecting to double every 20 years due to population aging. ${ }^{1} A D$ is known to have a long preclinical phase ${ }^{2}$ during which its pathophysiological processes could be detected by molecular and neuroimaging biomarkers. ${ }^{3}$

One of the best-established molecular biomarkers in dementia is the "AD signature" in cerebrospinal fluid (CSF), a decreased concentration of amyloid-beta peptide $\left(A \beta_{1}\right.$ 42) with increased total tau protein (t-Tau) and hyperphosphorylated tau $(\mathrm{p}-\mathrm{Tau}){ }^{4}$ This CSF profile has been already incorporated as supporting diagnostic criteria in international guidelines. ${ }^{4,5}$ Most research efforts are directed towards defining its level of accuracy in determining AD pathology as the underlying etiology of dementia and its power to predict conversion rates from mild cognitive impairment (MCl) or even asymptomatic (preclinical) stages to $A D,{ }^{4}$

Correspondence: Márcia Radanovic, Departamento de Psiquiatria, Universidade de Sao Paulo, Rua Dr. Ovidio Pires de Campos, 785, CEP 05403-010, Sao Paulo, SP, Brazil.

E-mail: marciaradanovic @gmail.com

Submitted Oct 03 2018, accepted Feb 05 2019, Epub May 302019. as a means to contribute to the differential diagnosis of dementia. ${ }^{6-8}$

It has been reported that levels of these biomarkers correlate with cognitive performance, especially in patients within the MCl-AD continuum, ${ }^{9,10}$ but this association is still controversial, ${ }^{11}$ and it is unclear whether this correlation is also found in non-AD dementias.

Our study aimed to correlate CSF levels of $A \beta_{1-42}$, t-Tau, p-Tau, and $A \beta_{1-42} / p$-Tau ratio with cognitive performance in healthy and cognitively impaired subjects $(\mathrm{MCl}$, $A D$, and non-AD dementias), starting from clinical diagnoses. To the best of our knowledge, no similar studies have been conducted in Brazilian populations.

\section{Methods \\ Participants}

Participants were recruited from a cohort of older adults who are regularly followed up at a university-based

How to cite this article: Radanovic M, Oshiro CA, Freitas TQ, Talib LL, Forlenza OV. Correlation between CSF biomarkers of Alzheimer's disease and global cognition in a psychogeriatric clinic cohort. Braz J Psychiatry. 2019;41:479-484. http://dx.doi.org/10.1590/15164446-2018-0296 
psychogeriatric clinic in São Paulo, Brazil. This facility receives patients referred from other hospitals due to suspected cognitive decline and those spontaneously seeking medical attention related to cognitive complaints or worries about developing dementia (for instance, individuals with one or more relatives who experienced cognitive decline in the old age). All participants were interviewed and evaluated by a multidisciplinary team of psychiatrists, neurologists, geriatricians, neuropsychologists, and occupational therapists. Clinical history and general and neurological examinations were performed before the cognitive diagnosis, which was obtained through a full neuropsychological and functional assessment that included the Fuld object memory evaluation (FOME), ${ }^{12}$ the Rivermead Behavioral Memory Test (RBMT), ${ }^{13}$ tasks A and B of the Trail Making Test (TMT), ${ }^{14}$ the Revised Wechsler Adult Intelligence Scale Vocabulary and Block Design subtests, ${ }^{15}$ and the Informant Questionnaire on Cognitive Decline in the Elderly (IQCODE).$^{16}$ Depressive symptoms were ruled out through the 21-item Hamilton Depression Rating Scale, ${ }^{17}$ with euthymia considered for scores $<8$. The Mini-Mental State Examination (MMSE) ${ }^{18,19}$ was also administered. All participants were screened for treatable causes of dementia (complete blood count, liver enzymes, serum vitamin $B_{12}$, HIV serology, VDRL, and kidney and thyroid function), as well as by neuroimaging (MRI) studies.

Exclusion criteria for all participants were: a) history or current neurological and/or psychiatric comorbidities (including major depression) which might lead to inaccurate cognitive assessment; b) uncompensated systemic diseases; and c) recent introduction or dose adjustment of medications that interfere with cognitive performance.

After the selection process, 208 participants were divided into four groups: $A D, M C l$, other dementias (OD), and control. Eighty-two participants were clinically diagnosed with $\mathrm{MCl}$ and 46 with $\mathrm{AD}$ according to the National Institute on Aging-Alzheimer's Association (NIA-AA) criteria. ${ }^{4,20}$ Twenty-six participants were diagnosed with non-AD dementias; frontotemporal lobar degeneration (FTLD) was most prevalent, and was diagnosed according to the Frontotemporal Dementia Consortium (FTDC) revised criteria. $^{21}$ Fifty-four participants with no evidence of cognitive impairment nor of any psychiatric disorders at the time of evaluation were defined as controls. Neither MMSE scores nor CSF biomarkers were used as diagnostic criteria for any groups.

\section{Cerebrospinal fluid analyses}

All participants underwent morning lumbar puncture in the L3/L4 or L4/L5 intervertebral space, using a 23-gauge needle. CSF samples containing $12-15 \mathrm{~mL}$ each were collected into polypropylene tubes, centrifuged at 3,200 $\times g$ for 10 minutes at $4{ }^{\circ} \mathrm{C}$, split into $0.5-\mathrm{mL}$ aliquots in cryotubes (Sarstedt), immediately frozen and stored at $-80^{\circ} \mathrm{C}$ until analysis. No samples were thawed or refrozen. Concentrations of $A \beta_{1-42}, t-T a u$, and $p$-Tau were then measured in duplicate using the INNO-BIA AlzBio3 immunoassay kit (Innogenetics, Ghent, Belgium).

A suspension of microspheres carrying the capturing antibodies (AT120, AT270, and 4D7A3 for t-Tau, p-Tau, and $A \beta_{1-42}$, respectively) was added to a pre-wetted filter plate with a wash buffer. A mixture of $75 \mu \mathrm{L}$ of CSF or standards along with biotinylated detection monoclonal antibodies designed for each of the capturing antibodies (HT7 for t-Tau and p-Tau and 3D6 for $A \beta_{1-42}$ ) was then added to the plate and incubated overnight in the dark. The plate was then washed, a detection conjugate (phycoerythrin-labeled streptavidin) added, and incubated for 1 hour at room temperature. The plate was washed again and, after addition of a reading solution (phosphatebuffered saline), the assay was finally analyzed in a Luminex 100IS platform (Luminex, Austin, TX, USA).

Standard curves were constructed for each biomarker by a sigmoidal curve-fitting method, and the mean fluorescence values for the duplicate CSF samples were used to determine the concentration of $A \beta_{1-42}, \mathrm{t}-\mathrm{Tau}$, and p-Tau.

\section{Statistical analyses}

Demographic and clinical characteristics were analyzed by comparison of means \pm standard deviation or frequency of distribution among groups. One-way analysis of variance (ANOVA) was used for normally distributed variables, and the Kruskal-Wallis test for nonparametric variables. For the categorical variable sex, we used the chi-square test. Analyses to assess betweengroup differences were followed by Student's t-tests. Pearson correlation coefficients were calculated to determine the association between MMSE scores and biomarkers, adjusted for age and education.

In all tests, we considered p-values below 0.05 as statistically significant.

\section{Standard protocols, registrations, and patient consent}

The institutional ethics committee of Hospital das Clínicas, Faculdade de Medicina, Universidade de São Paulo, and a national ethics committee (Plataforma Brasil) approved this study. All subjects or their legal guardians provided written consent prior to enrollment in the assessment protocol.

\section{Data availability statement}

All raw data not published within the article are publicly available through the Open Science Framework (OSF) at doi:10.17605/OSF.IO/WXFTG.

\section{Results}

There were no statistically significant differences among groups regarding age and sex. Controls had significantly more years of schooling than the other groups. They also had significantly higher MMSE scores, as expected. Regarding CSF biomarkers, patients in the AD group had significantly lower levels of $A \beta_{1-42}$, higher levels of $t-T a u$, and a lower $A \beta_{1-42} / P$-tau ratio when compared to the control and $\mathrm{MCl}$, but not $\mathrm{OD}$, groups; P-tau levels were highest in the $A D$ group as compared to all remaining groups (Table 1). 
Table 1 Demographic characteristics, cognitive performance, and concentrations of biomarkers $\left(A \beta_{1-42}\right.$, t-Tau, and $\left.p-T a u\right)$ in cerebrospinal fluid according to baseline diagnosis

\begin{tabular}{|c|c|c|c|c|c|c|}
\hline Variable & $C(n=54)$ & $\mathrm{MCl}(\mathrm{n}=82)$ & $A D(n=46)$ & OD $(n=26)$ & $\mathrm{p}$ & $\begin{array}{l}\text { Multiple comparison } \\
\qquad<<0.05\end{array}$ \\
\hline Age (years) & $71.3(5.0)$ & $72.8(6.2)$ & $73.4(7.2)$ & $70.8(6.3)$ & 0.220 & $\mathrm{~N} / \mathrm{A}$ \\
\hline Schooling (years) & $11.5(5.8)$ & $8.9(5.4)$ & $8.7(4.8)$ & $8.4(5.3)$ & 0.035 & $\mathrm{C} \neq \mathrm{MCl}, \mathrm{AD} \& \mathrm{OD}$ \\
\hline Sex, M/F & $35 / 19$ & $51 / 31$ & $24 / 22$ & $21 / 5$ & 0.114 & $N / A$ \\
\hline MMSE & 27.4 (3.7) & $26.1(3.2)$ & $22.5(5.0)$ & $22.9(6.0)$ & $<0.0001$ & $\mathrm{C} \neq \mathrm{MCl}, \mathrm{AD} \& \mathrm{OD}$ \\
\hline$A \beta_{1-42}(\mathrm{pg} / \mathrm{mL})$ & $461.6(170.5)$ & $450.0(178.6)$ & 365.3 (126.3) & $434.0(182.3)$ & 0.022 & $\mathrm{AD} \neq \mathrm{C} \& \mathrm{MCl}$ \\
\hline t-Tau (pg/mL) & $91.1(57.7)$ & $103.1(73.8)$ & $133.9(90.8)$ & $100.2(64.4)$ & 0.029 & $\mathrm{AD} \neq \mathrm{C} \& \mathrm{MCl}$ \\
\hline p-Tau (pg/mL) & $46.8(30.9)$ & $45.6(29.0)$ & 64.6 (39.3) & $35.9(14.6)$ & 0.001 & $A D \neq C, M C l \& O D$ \\
\hline$A \beta_{1-42} / p-T a u$ & $13.4(8.6)$ & $13.3(8.9)$ & $8.3(7.4)$ & $13.6(7.9)$ & 0.006 & $\mathrm{AD} \neq \mathrm{C} \& \mathrm{MCl}$ \\
\hline
\end{tabular}

Data presented as mean (standard deviation), unless otherwise specified.

$\mathrm{A} \beta_{1-42}=$ amyloid-beta peptide; $\mathrm{AD}=$ Alzheimer's disease $; \mathrm{C}=$ controls; $\mathrm{MCl}=$ mild cognitive impairment; MMSE = Mini-Mental State Examination; N/A = not applicable; OD = other dementias; $p$-Tau $={ }^{181}$ Thr-phosphorylated-tau; $t$-Tau $=$ total tau.

Table 2 Correlations $(r)$ between biomarkers in cerebrospinal fluid and MMSE scores for each diagnostic group

\begin{tabular}{|c|c|c|c|c|c|}
\hline MMSE vs. & Total sample & Control & $\mathrm{MCl}$ & $A D$ & OD \\
\hline$A \beta_{1-42}$ & $\begin{array}{c}0.242 \\
p=0.0006\end{array}$ & $\begin{array}{c}0.211 \\
p=0.132\end{array}$ & $\begin{array}{c}0.247 \\
p=0.030\end{array}$ & $\begin{array}{c}-0.145 \\
p=0.358\end{array}$ & $\begin{array}{c}0.440 \\
p=0.027\end{array}$ \\
\hline t-Tau & $\begin{array}{l}-0.325 \\
p<0.0001\end{array}$ & $\begin{array}{c}-0.284 \\
p=0.043\end{array}$ & $\begin{array}{c}-0.241 \\
p=0.036\end{array}$ & $\begin{array}{c}-0.282 \\
p=0.070\end{array}$ & $\begin{array}{c}-0.665 \\
p=0.0003\end{array}$ \\
\hline p-Tau & $\begin{array}{c}-0.247 \\
p=0.0005\end{array}$ & $\begin{array}{c}-0.206 \\
p=0.141\end{array}$ & $\begin{array}{c}-0.092 \\
p=0.426\end{array}$ & $\begin{array}{c}-0.343 \\
p=0.026\end{array}$ & $\begin{array}{c}-0.540 \\
p=0.005\end{array}$ \\
\hline $\mathrm{A} \beta_{1-42} / \mathrm{p}-\mathrm{Tau}$ & $\begin{array}{c}0.277 \\
p=0.0001\end{array}$ & $\begin{array}{c}0.207 \\
p=0.140\end{array}$ & $\begin{array}{c}0.137 \\
p=0.233\end{array}$ & $\begin{array}{c}0.176 \\
p=0.262\end{array}$ & $\begin{array}{c}0.597 \\
p=0.001\end{array}$ \\
\hline
\end{tabular}

$\mathrm{A} \beta_{1-42}=$ amyloid-beta peptide; $\mathrm{AD}=$ Alzheimer's disease; $\mathrm{p}$-Tau $={ }^{181}$ Thr-phosphorylated-tau; $\mathrm{t}$-Tau $=$ total tau; $\mathrm{MCl}=$ mild cognitive impairment; MMSE = Mini-Mental State Examination; OD = other dementias.

MMSE scores correlated weakly with all CSF biomarkers in the total sample. When considering correlations across diagnostic groups, $A \beta_{1-42}$ levels and MMSE scores correlated weakly in the $\mathrm{MCl}$ group, and moderately in the OD group. t-Tau levels showed a mild inverse correlation with MMSE scores in the control and $\mathrm{MCl}$ groups, and this correlation was moderate/strong in the OD group. $\mathrm{p}$-Tau levels correlated with MMSE scores in the AD (weak correlation) and OD (moderate correlation) groups. Finally, the $A \beta_{1-42} / p$-Tau ratio had a moderate to strong correlation with MMSE scores in the OD group (Table 2 and Figure 1).

\section{Discussion}

Measurement of biomarkers in CSF is a valuable tool in clinical practice, and its use is becoming increasingly widespread as a means of determining the underlying pathology in dementias of atypical presentation ${ }^{22}$ and in predicting the risk of developing dementia in $\mathrm{MCl}$ and asymptomatic older adults. ${ }^{23}$ Moreover, CSF biomarker levels are useful in the differential diagnosis of dementia. ${ }^{24}$ Currently, $A \beta_{1-42}$, t-Tau, $p-T a u$, and combined ratios thereof are the best-studied CSF biomarkers; $A \beta_{1-42}$ is considered a marker of amyloid deposition, whereas $\mathrm{t}$-Tau and $\mathrm{p}$-Tau are considered markers of neuronal injury. ${ }^{25}$

However, the reliability of these CSF biomarkers in reflecting the rate of cognitive decline over the course of dementing illnesses has yet to be determined. Studies focusing on this issue have reached contradictory results, ${ }^{11,26}$ and most recent studies have focused on the association between the "AD-signature" and impairments in specific cognitive domains, such as memory. ${ }^{27}$

Our results show that patterns of CSF biomarker levels differed in each group, with the $A D$ group exhibiting significantly higher levels of $p$-Tau and t-Tau, lower $A \beta_{1-42}$ levels, and, consequently, a lower $A \beta_{1-42} / p-T a u$ ratio, as expected. ${ }^{4}$ In the AD group, cognitive performance correlated only with $p$-Tau levels. Increased levels of $\mathrm{p}$-Tau are considered to strengthen sensitivity for differential diagnosis of $A D^{24}$ and were capable of differentiating $A D$ from $O D$ in our sample. Seppälä et al. ${ }^{28}$ described a correlation between decreasing rates of $p$-Tau and MMSE scores in $A D$, which was interpreted as a sign of progressive neuronal loss. Rolstad et al. ${ }^{9}$ described a more significant impact of CSF $A \beta_{1-42}$ levels in episodic memory and visuospatial abilities, while t-Tau levels correlated better with episodic memory in patients with dementia. In 2013, Rolstad et al. ${ }^{26}$ replicated their study and confirmed that $A \beta_{1-42}$ and t-Tau levels in CSF were associated with semantic and working memory performance, with the effect size of $\mathrm{t}$-Tau levels being larger than that of $A \beta_{1-42}$. Rami et al. ${ }^{27}$ also found a correlation between memory performance and CSF $A \beta_{1-42}$ levels in AD. However, negative reports have also been published. ${ }^{11,29}$ More recently, Mandecka et al. ${ }^{30}$ described an association between the severity of verbal memory impairment and the degree of CSF abnormalities in AD.

In the $\mathrm{MCl}$ group, there was a statistically significant negative correlation between MMSE scores and t-Tau levels in CSF, as well as a positive correlation with $A \beta_{1-42}$ levels. Memory performance has been associated with higher t-Tau and $\mathrm{p}$-Tau levels in $\mathrm{MCl}$ populations, ${ }^{27}$ 

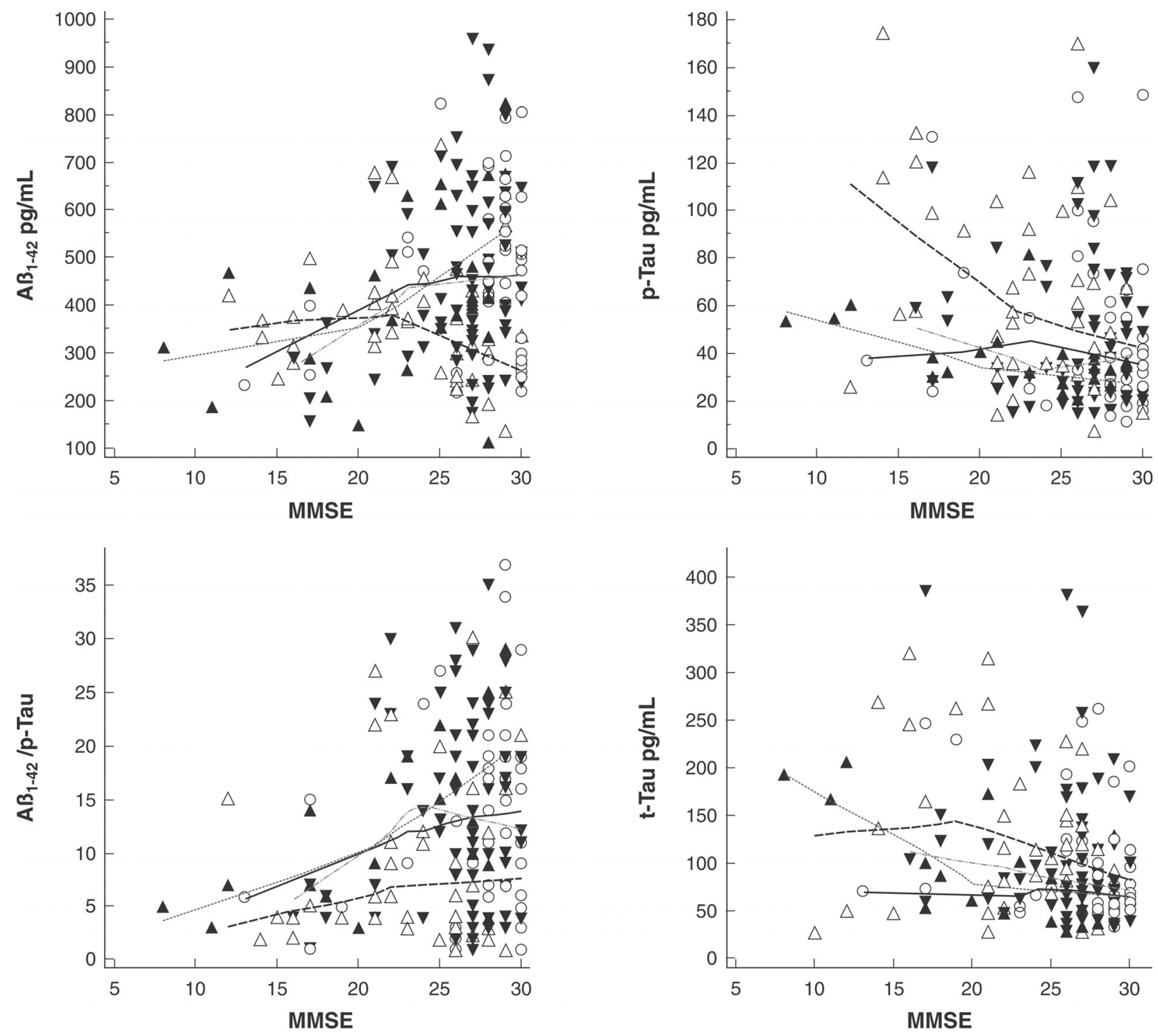

$$
\text { Diagnosis } \bigcirc \text { Controls } \quad \nabla \mathrm{MCl} \triangle \mathrm{AD} \quad \triangle \mathrm{OD}
$$

Figure 1 Correlations between cerebrospinal fluid biomarkers and MMSE scores in each diagnostic group. $A \beta_{1-42}=$ amyloidbeta peptide; $A D=$ Alzheimer's disease; $p$-Tau $={ }^{181} \mathrm{Thr}$-phosphorylated-tau; $\mathrm{t}$-Tau = total tau; $\mathrm{MCl}=$ mild cognitive impairment; MMSE = Mini-Mental State Examination; OD = other dementias.

as well as with $A \beta_{1-42}$ levels. ${ }^{30,31}$ Rolstad et al..$^{9,26}$ found a more widespread pattern of correlation across all cognitive domains. Nathan et al. ${ }^{32}$ recently described a relationship between sustained attention and memory performance and higher t-Tau and p-Tau levels in CSF. Such findings, in association with those described for AD patients, led to the currently accepted notion that, in the continuum from healthy aging to $A D, C S F$ biomarker levels can reflect cognitive decline. This association, however, seems to obey a temporal pattern, in which cognitive performance first correlates with $A \beta_{1-42}$ levels, then with t-Tau and $p$-Tau levels, and finally becomes independent from biomarkers in fully developed dementia. Thus, the combination of neuropsychological examination and analysis of the CSF biomarker profile seems promising in predicting conversion to dementia, ${ }^{33,34}$ especially AD.

In the control group, we found a negative correlation between $\mathrm{t}$-Tau levels and cognitive performance. Studies focusing on CSF biomarker levels in cognitively healthy subjects have demonstrated that a proportion of such subjects exhibit both evidence of amyloid deposition (as indicated by low CSF levels of $A \beta_{1-42}$ ) and/or neurodegeneration (as shown by high levels of t-Tau and $\mathrm{p}$-Tau). This proportion tends to increase through the aging process and is affected by presence of the APOE4 genotype. ${ }^{25,35}$ Subjects who show evidence of neurodegeneration, but not of amyloid deposition, known as the "suspected non-Alzheimer pathology" (SNAP) 
category, ${ }^{36}$ represent those who not display underlying $A D$ pathology and would probably develop non-AD dementias. ${ }^{35}$ Studies in cognitively preserved individuals have disclosed an association between $A \beta_{1-42}$ levels in CSF and episodic, semantic, and working memory performance, although most cases assessed individuals with subjective memory complaints (SMC) ${ }^{9,27,30}$ Only one study ${ }^{26}$ reported an impact of t-Tau levels in executive functions in SMC. As noted in the Methods section, although our control group was composed of cognitively healthy subjects (as defined through standard cognitive and functional assessment), they might be considered as an SMC group, as they presented with mild memory complaints ("forgetfulness").

Lastly, the group in which the strongest correlations between CSF levels and cognition occurred was the nonAD dementia group, where most patients had FTLD (either behavioral variant or primary progressive aphasia). We found only two studies describing a correlation between CSF $A \beta_{1-42}$ levels and general cognitive function ${ }^{37}$ and memory performance (both learning and recall) ${ }^{38}$ in FTD. Tau levels have already been associated with survival rates in FTD, ${ }^{39}$ but we did not find any reports of association between neuronal injury biomarkers and cognition in this population.

In summary, we observed that all CSF biomarkers correlated to some extent with overall cognitive performance when considering the sample as a whole. A more thorough analysis revealed that $A \beta_{1-42}$ levels were associated with cognitive performance in the $\mathrm{MCl}$ and OD groups. Several studies have established this association in $\mathrm{MCl}$, although focusing on isolated cognitive domains. ${ }^{10,28,29}$ Tau levels correlated with cognitive performance in the control, $\mathrm{MCl}$, and non- $\mathrm{AD}$ dementia groups. As already noted, t-Tau levels are markers of neuronal injury, which may explain why they correlate more consistently with diagnostic status in earlier (oligosymptomatic) stages of cognitive decline, reaching a "ground effect" once the dementia is fully developed (in AD). p-Tau levels correlated more specifically with cognition in the $A D$ group, which probably reflects the greater specificity of this biomarker for $A D$ diagnosis. ${ }^{25}$ Recently, Koychev et al. ${ }^{40}$ described a pattern of correlation between CSF levels of $t-T a u$ (but not of $A \beta_{1-42}$ ) and cognitive decline assessed through the ADAS-Cog, which is also suitable for measuring global cognition. Bouallègue et al. ${ }^{41}$ reported a similar correlation (CSF t-Tau, but not $A \beta_{1-42}$, levels vs. cognitive performance) in a crosssectional cohort assessed with the ADAS-Cog, MMSE, and CDR; longitudinal observation found that t-Tau levels were associated with cognitive worsening as measured by a decrease in MMSE scores only (and not in ADASCog or CDR), while $p$-Tau levels were associated with baseline diagnosis (control, $\mathrm{MCl}$, or $\mathrm{AD}$ ). However, Joachim et al. ${ }^{42}$ found a significant decrease in cognitive performance using the ADAS-Cog in patients with $\mathrm{MCl}$ who had lower levels of $A \beta_{1-42}$ and higher levels of $t-T a u$, despite treatment with acetylcholinesterase inhibitors. Such heterogeneous results raise some questions regarding the optimal outcome measure for primary clinical settings, as the association between CSF biomarker levels and global measures of cognition seems to reach a "steady state" close to the initial stages of fully developed AD.

Finally, t-Tau levels and t-Tau/A $A \beta_{1-42}$ ratios are considered useful for differential diagnosis between $A D$ and non-AD dementias, especially FTD, although their accuracy is highly variable across studies. t-Tau concentrations are significantly higher in FTD than in controls, but significantly lower than in AD. p-Tau concentrations show good capacity to differentiate AD cases from FTD. ${ }^{43}$ Unfortunately, the lack of data on the association between cognitive performance and CSF biomarkers in FTD hampers comparison with our data.

The main limitations of this study are its cross-sectional design, which is not the best suited to verify changes in biomarker levels across the natural history of disease, and the gact that the $\mathrm{MCl}$ group was not subdivided into amnestic (AD-MCI) and nonamnestic subjects, as these two populations are prone to developing dementia of different etiologies. Regarding the use of the MMSE (a screening test) as the cognitive outcome measure, it was our purpose to verify the association of CSF biomarkers with global cognition rather than with specific domains, in a study design with both an ecological and practical focus. We understand that further studies using more refined global cognitive measures, larger samples (especially for non-AD dementias), and, most of all, a longitudinal design are necessary to establish the mechanisms that ultimately lead to clinically significant neuronal injury, which is pivotal in developing diseasemodification therapies. However, to the best of our knowledge, this is the first study to address such correlations in the Brazilian population.

\section{Acknowledgements}

Study funded by Associação Beneficente Alzira Denis Hertzog da Silva (ABADHS), Instituto Nacional de Biomarcadores em Neuropsiquiatria (INBION), Fundação de Amparo à Pesquisa do Estado de São Paulo (FAPESP; Projects 09/52825-8, 2014/14211-6, 2016/01302-9), and Conselho Nacional de Desenvolvimento Científico e Tecnológico (CNPq; Projects 442795/2014-9 and 466625/2014-6).

\section{Disclosure}

The authors report no conflicts of interest.

\section{References}

1 Alzheimer's Association. 2015 Alzheimer's disease facts and figures. Alzheimers Dement. 2015;11:322-84.

2 Jack Cr Jr, Albert MS, Knopman DS, McKhann GM, Sperling RA, Carrillo $\mathrm{MC}$, et al. Introduction to the recommendations from the National Institute on Aging-Alzheimer's Association workgroups on diagnostic guidelines for Alzheimer's disease. Alzheimers Dement. 2011;7:257-62.

3 Jack Jr. CR, Bennett DA, Blennow K, Carrillo MC, Dunn B, Haeberlein $\mathrm{SB}$, et al. NIA-AA research framework: toward a biological definition of Alzheimer's disease. Alzheimers Dement. 2018;14:535-62.

4 Albert MS, DeKosky ST, Dickson D, Dubois B, Feldman HH, Fox NC, et al. The diagnosis of mild cognitive impairment due to Alzheimer's 
disease: recommendations from the National Institute on AgingAlzheimer's Association workgroups on diagnostic guidelines for Alzheimer's disease. Alzheimers Dement. 2011;7:270-9.

5 Custodio N, Wheelock A, Thumala D, Slachevsky A. Dementia in Latin America: epidemiological evidence and implications for public policy. Front Aging Neurosci. 2017;9:221.

6 Hermann P, Romero C, Schmidt C, Reis C, Zerr I. CSF biomarkers and neuropsychological profiles in patients with cerebral small-vessel disease. PLoS One. 2014;9:e105000.

7 van Steenoven I, Aarsland D, Weintraub D, Londos E, Blanc F, van der Flier WM, et al. Cerebrospinal fluid Alzheimer's disease biomarkers across the spectrum of Lewy body diseases: results from a large multicenter cohort. J Alzheimers Dis. 2016;54:287-95.

8 Oeckl P, Steinacker P, Feneberg E, Otto M. Neurochemical biomarkers in the diagnosis of frontotemporal lobar degeneration: an update. J Neurochem. 2016;138:184-92.

9 Rolstad S, Berg Al, Bjerke M, Blennow K, Johansson B, Zetterberg H, et al. Amyloid- $\beta_{42}$ is associated with cognitive impairment in healthy elderly and subjective cognitive impairment. J Alzheimers Dis. 2011; 26:135-42.

10 Bertens D, Knol DL, Scheltens P, Visse PJ; Alzheimer's Disease Neuroimaging Initiative. Temporal evolution of biomarkers and cognitive markers in the asymptomatic, $\mathrm{MCl}$, and dementia stage of Alzheimer's disease. Alzheimers Dement. 2015;11:511-22.

11 Williams JH, Wilcock GK, Seeburger J, Dallob A, Laterza O, Potter $W$, et al. Non-linear relationships of cerebrospinal fluid biomarker levels with cognitive function: an observational study. Alzheimers Res Ther. 2011;3:5

12 Fuld PA. Guaranteed stimulus-processing in the evaluation of memory and learning. Cortex. 1980;16:225-71.

13 Wilson BA, Baddeley AD, Cockburn JM. The Rivermead behavioural memory test. 2nd ed. Suffolk: Thames Valley Company; 1991.

14 Army individual test battery. Manual of directions and scoring Washington: War Department, Adjunt General's Office; 1944.

15 Wechsler DI. Examiner's manual: Wechsler adult intelligence scalerevised. New York: Psychological Corporation; 1981.

16 Jorm AF, Jacomb PA. The Informant Questionnaire on Cognitive Decline in the Elderly (IQCODE): socio-demographic correlates, reliability, validity and some norms. Psychol Med. 1989;19:1015-22.

17 Hamilton M. A rating scale for depression. J Neurol Neurosurg Psychiatry. 1960;23:56-62.

18 Folstein MF, Folstein SE, McHugh PR. "Mini -Mental State". A practical method for grading the cognitive state of patients for the clinician. J Psychiatr Res.1975;12:189-98.

19 Kochann R, Cerveira MO, Godinho C, Camozzato A, Chaves ML. Evaluation of the Mini-Mental State Examination scores according to different age and education strata, and sex, in a large Brazilian healthy sample. Dement Neuropsychol. 2009;3:88-93.

20 McKhann GM, Knopman DS, Chertkow H, Hyman BT, Jack CR Jr, Kawas $\mathrm{CH}$, et al. The diagnosis of dementia due to Alzheimer's disease: recommendations from the National Institute on AgingAlzheimer's Association workgroups on diagnostic guidelines for Alzheimer's disease. Alzheimers Dement. 2011;7:263-9.

21 Rascovsky K, Hodges JR, Knopman D, Mendez MF, Kramer JH, Neuhaus J, et al. Sensitivity of revised diagnostic criteria for the behavioural variant of frontotemporal dementia. Brain. 2011;134: 2456-77.

22 Simonsen AH, Herukka Sk, Andreasen N, Baldeiras I, Bjerke M, Blennow K, et al. Recommendations for CSF AD biomarkers in the diagnostic evaluation of dementia. Alzheimers Dement. 2017;13: 274-84.

23 Vos SJ, Verhey F, Frölich L, Kornhuber J, Wiltfang J, Maier W, et al. Prevalence and prognosis of Alzheimer's disease at the mild cognitive impairment stage. Brain. 2015;138:1327-38.

24 Llorens F, Schmitz M, Ferrer I, Zerr I. CSF biomarkers in neurodegenerative and vascular dementias. Prog Neurobiol. 2016;138-140: 36-53.

25 Jack CR Jr, Wiste HJ, Weigand SD, Rocca WA, Knopman DS, Mielke $\mathrm{MM}$, et al. Age-specific population frequencies of cerebral $\beta$-amyloidosis and neurodegeneration among people with normal cognitive function aged 50-89 years: a cross-sectional study. Lancet Neurol. 2014;13:997-1005
26 Rolstad S, Berg Al, Bjerke M, Johansson B, Zetterberg $\mathrm{H}$, Wallin A. Cerebrospinal fluid biomarkers mirror rate of cognitive decline. J Alzheimers Dis. 2013;34:949-56.

27 Rami L, Fortea J, Bosch B, Solé-Padullés C, Lladó A, Iranzo A, et al. Cerebrospinal fluid biomarkers and memory present distinct associations along the continuum from healthy subjects to AD patients. J Alzheimers Dis. 2011;23:319-26.

28 Seppälä TT, Koivisto AM, Hartikainen P, Helisalmi S, Soininen $H$ Herukka SK. Longitudinal changes of CSF biomarkers in Alzheimer's disease. J Alzheimers Dis. 2011;25:583-94.

29 Spies PE, Claassen JAHR, Slats D, Olde Rikkert MGM, Verbeek M, Kessels RPC. Cerebrospinal fluid tau and amyloid $\beta$ proteins do not correlate with cognitive functioning in cognitively impaired memory clinic patients. CNS Spectrums. 2010;15:588-93.

30 Mandecka M, Budziszewska M, Barczak A, Pepłońska B, Chodakowska-Żebrowska M, Filipek-Gliszczyńska A, et al. Association between cerebrospinal fluid biomarkers for Alzheimer's disease, APOE genotypes and auditory verbal learning task in subjective cognitive decline, mild cognitive impairment, and Alzheimer's disease. J Alzheimers Dis. 2016;54:157-68.

31 Kandel BM, Avants BB, Gee JC, Arnold SE, Wolk DA; Alzheimer's Disease Neuroimaging Initiative 1. Neuropsychological testing predicts cerebrospinal fluid amyloid- $\beta$ in mild cognitive impairment. J Alzheimers Dis. 2015;46:901-12.

32 Nathan PJ, Lim YY, Abbot R, Galluzzi S, Marizzoni M, Babiloni C, et al. on behalf of the PharmaCog Consortium. Association between CSF biomarkers, hippocampal volume and cognitive function in patients with amnestic mild cognitive impairment $(\mathrm{MCl})$. Neurobiol Aging. 2017;53:1-10.

33 Eckerström C, Olsson E, Klasson N, Berge J, Nordlund A, Bjerke M, et al. Multimodal prediction of dementia with up to 10 years follow up: the Gothenburg MCl study. J Alzheimers Dis. 2015;44:205-14.

34 Mazzeo S, Santangelo R, Bernasconi MP, Cecchetti G, Fiorino A Pinto $P$, et al. Combining cerebrospinal fluid biomarkers and neuropsychological assessment: a simple and cost-effective algorithm to predict the progression from mild cognitive impairment to Alzheimer's disease dementia. J Alzheimers Dis. 2016;54: 1495-508.

35 Toledo JB, Zetterberg H, Van Harten A, Glodzik L, Martinez-Lage P Bocchio-Chiavetto $L$, et al. Alzheimer's disease cerebrospinal fluid biomarker in cognitively normal subjects. Brain. 2015;138:2701-15.

36 Jack CR Jr, Knopman DS, Chételat G, Dickson D, Fagan AM, Frison $\mathrm{GB}$, et al. Suspected non-Alzheimer disease pathophysiologyconcept and controversy. Nat Rev Neurol. 2016;12:117-24.

37 Koedam EL, van der Vlies AE, van der Flier WM, Verwey NA, Koene $T$, Scheltens $P$, et al. Cognitive correlates of cerebrospinal fluid biomarkers in frontotemporal dementia. Alzheimers Dement. 2013;9: 269-75.

38 Körtvélyessy O, Gukasjan A, Sweeney-Reed CM, Heinze HJ, Thurner L, Bittner DM. Progranulin and amyloid- $\beta$ levels: relationship to neuropsychology in frontotemporal and Alzheimer's disease. J Alzheimers Dis. 2015;46:375-80.

39 Borroni B, Benussi A, Cosseddu M, Archetti S, Padovani A. Cerebrospinal fluid tau levels predict prognosis in non-inherited frontotemporal dementia. Neurodegener Dis. 2014;13:224-9.

40 Koychev I, Gunn RN, Firouzian A, Lawson J, Zamboni G, Ridha B, et al. PET Tau and amyloid- $\beta$ burden in mild Alzheimer's disease: divergent relationship with age, cognition, and cerebrospinal fluid biomarkers. J Alzheimers Dis. 2017;60:283-93.

41 Ben Bouallègue F, Mariano-Goulart D, Payoux P; Alzheimer's Disease Neuroimaging Initiative (ADNI). Comparison of CSF markers and semi-quantitative amyloid PET in Alzheimer's disease diagnosis and in cognitive impairment prognosis using the ADNI-2 database. Alzheimers Res Ther. 2017;9:32.

42 Joachim LK, Frölich L, Rüther E, Wiltfang J, Maier W, Kornhuber J, et al. Correlation of CSF- and MRI- biomarkers and progression of cognitive decline in an open label $\mathrm{MCl}$ trial. J Prev Alzheimers Dis. 2018;5:202-6.

43 Ferreira D, Perestelo-Pérez L, Westman E, Wahlund LO, Sarría A Serrano-Aguilar P. Meta-Review of CSF core biomarkers in Alzheimer's Disease: the state-of-the-art after the new revised diagnostic criteria. Front Aging Neurosci. 2014;6:47. 